ẼESTI NSV TEADUSTE AK̇ADEEMIA TOIMETISED, 27, KOIDE

FUOSIKA * MATEMAATIKA. 1978, NR. 2

ИЗВЕСТИЯ АКАДЕМИИ НАУК ЭСТОНСКОИ ССР. ТОМ 27 ФНЗИКА * МАТЕМАТИҚА, 1978, № 2

А. ВОЛЬДЕК|, Г. СКРЯБИНА

\title{
ТЕОРИЯ ЛИНЕИНОИ АСИНХРОННОЙ МАШИНЫ С УЧЕТОМ ПРОДОЛЬНОГО И ПОПЕРЕЧНОГО КРАЕВЫХ ЭФФЕКТОВ
}

\section{Постановка задачи}

Линейные асинхронные машины (ЛАМ) перспективны в качестве тяговых двигателей для высокоскоростного наземного транспорта. По теории ЛАМ опубликовано много статей. Однако среди них почти нет работ, в которых одновременно учитывались бы продольные и поперечные краевые эффекты, обусловленные конечной длиной и конечной шириной машины, а также работ по теории ЛАМ с двуслойными обмотками. Исключение составляют работы К. Оберретля $\left[{ }^{1,2}\right]$. Однако теория, созданная К. Оберретлем, излишне усложнена. К тому же в ней не корректно учитывается влияние выступающих боковых сторон вторичной шины, которые предполагаются расположенными между ферромагнитными сердечниками. Ряды в выражениях К. Оберретля сходятся плохо. В указанной работе имеются и другие существенные недостатки.

Ниже излагается разработанная авторами теория ЛАМ, учитывающая влияние конечных продольных и поперечных размеров машины и лишенная перечисленных недостатков.

Рассматривается плоская двусторонняя машина (рис. 1) с двуслойной обмоткой и наполовину заполненными пазами концевых зон. Чтобы результаты анализа можно было распространить на аналогичные машины с однослойной обмоткой и полностью заполненными пазами, вводится функция $g=1$ для двуслойной обмотки и $g=0$ для однослойной концентрической обмотки. Полученные результаты действительны также для односторонней машины при условии, что пространство $z<0$, $-c \leqslant y \leqslant c$ (рис. 1) заполнено ферромагнитным сердечником с магнитной проницаемостью $\mu=\infty$ и удельной электрической проводимостью $\gamma=0$.

Принимаются следующие допущения, которые в основном соответствуют допущениям $\left[{ }^{3}\right]$ :

1. Ферромагнитные сердечники лишены пазов и имеют $\mu_{\mathrm{c}}=\infty$ и $\gamma_{\mathrm{c}}=0$.

2. Для учета влияния пазов расчетный немагнитный зазор принимается равным $\delta^{\prime}=k_{\delta} \delta$, где $k_{\delta}-$ коэффициент зазора.

3. Реальная вторичная шина толщиной $\Delta$ и электропроводностью $\gamma_{\text {шा }}$ заменяется шиной толщиной $\delta^{\prime}$ с $\gamma^{\prime}=\left(\Delta \gamma_{\text {ш }}\right) / \delta^{\prime}$ и $\mu=\mu_{0}$. Это не приводит к существенным погрешностям, поскольку на практике обычно $\Delta$ меньше двойной эквивалентной глубины проникновения тока и $\tau / \delta \geqslant 7$. 

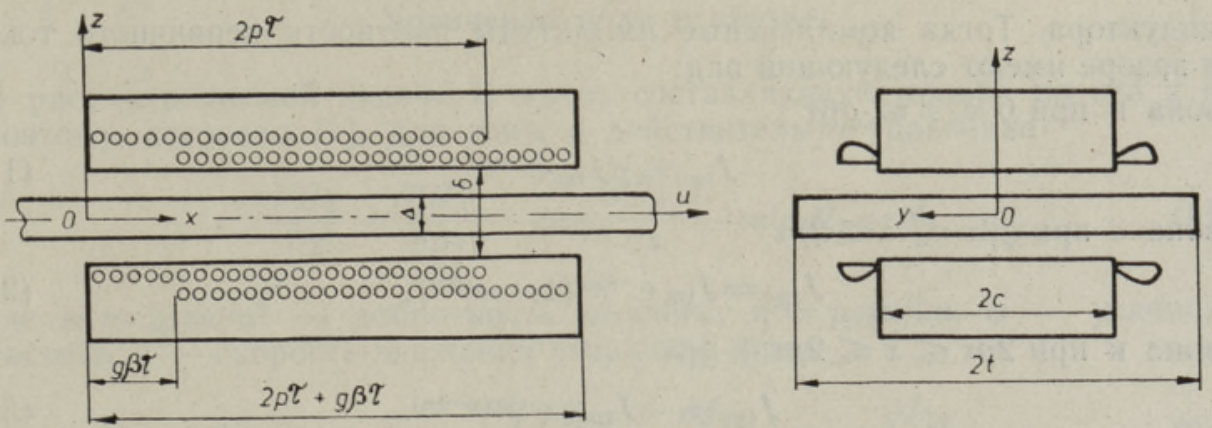

Рис. 1. Эскиз плоской ЛАМ с двуслойной обмоткой.
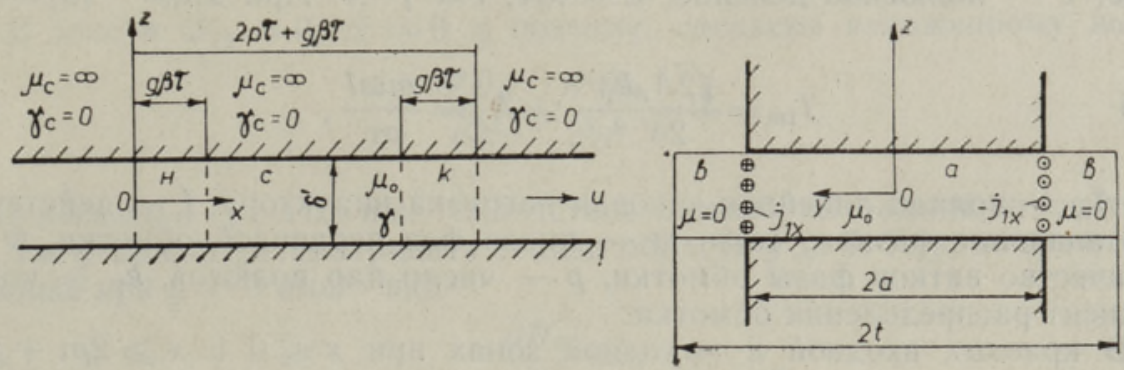

Рис. 2. Расчетная модель ЛАМ.

4. Ферромагнитные сердечники простираются вдоль координаты $x$ в обе стороны до бесконечности.

5. Для учета магнитного потока, выпучивающегося из зазора в боковых областях, принимается, что расчетная ширина сердечников $2 a=$ $=2 c+\delta^{\prime}\left[{ }^{4}\right]$.

6. Магнитная индукция за пределами сердечников в областях $|y| \geqslant a$ отсутствует.

7. Ток первичной обмотки распределен в зазоре равномерно по толщине $\delta^{\prime}$.

8. Токи лобовых ча̊стей обмотки сосредоточены в бесконечно тонких поверхностных слоях в зазоре между боковыми гранями сердечника (рис. 2).

9. В боковых областях шины в индуктированные э. д. с. отсутствуют, а вторичные токи распределены по законам постоянного тока. Формально это учитывается тем, что в этих областях шины полагается $\mu=0$, благодаря чему при $\mathbf{H} \neq 0$ индукция $\mathbf{B}=0$. тора.

10. Оси координат $x, y, z$ жестко связаны с сердечниками индук-

Согласно принятым допущениям, электромагнитное поле в зазоре машины распределено вдоль координаты $z$ равномерно и не зависит от нее, вследствие чего задача становится двумерной. Векторы напряженности магнитного поля $\mathbf{H}$, электрического поля $\mathbf{E}$ и плотности тока $\mathbf{j}$ имеют компоненты $\mathbf{H}\left(0,0, H_{z}\right), \mathbf{E}\left(E_{x}, E_{y}, 0\right), \mathbf{j}\left(j_{x}, j_{y}, 0\right)$ соответственно, которые являются функциями координат $x, y$ и времени $t$.

Учитывается только основная гармоника линейной токовой нагрузки 
индуктора. Тогда комплексные амплитуды плотности первичного тока в зазоре имеют следующий вид:

зона н при $0 \leqslant x \leqslant g \beta \tau$

$$
f_{1 y \mathrm{H}}=g J_{1 m} \mathrm{e}^{-i \alpha x},
$$

зона с при $g \beta \tau \leqslant x \leqslant 2 p \tau$

$$
J_{1 y c}=J_{1 m} \mathrm{e}^{-i \alpha x}\left(1-\mathrm{e}^{i \beta \pi}\right),
$$

зона к при $2 p \tau \leqslant x \leqslant 2 p \tau+g \beta \tau$

$$
J_{1 y \mathrm{\kappa}}=-J_{1 m \mathrm{~g}} \mathrm{e}^{-i(\alpha x-\beta \pi)} .
$$

Здесь $J_{1 m}=J_{1 m} \mathrm{e}^{i \Psi}-$ комплексная амплитуда плотности первичного тока в зазоре на концевых участках длиной $\beta \tau ; \beta-$ относительный шаг обмотки, равный в случае однослойной концентрической обмотки единице; $\tau$ - полюсное деление; $\alpha=\pi / \tau ; i=\sqrt{-1}$. При этом

$$
J_{1 m}=\frac{\sqrt{2} A_{\text {Л }} k_{\mathrm{p}}}{2 \delta^{\prime}}, \quad A_{\text {Л }}=\frac{m \omega I}{p \tau},
$$

где $A_{\text {л }}$ - полная линейная токовая нагрузка индуктора, $I$ - действующее значение фазного тока, $m$ - число фаз первичной обмотки, w количество витков фазы обмотки, $p-$ число пар полюсов, $k_{\mathrm{p}}-$ коэффициент распределения обмотки.

В краевых входной и выходной зонах при $x \leqslant 0$ и $x \geqslant 2 p \tau+g \beta \tau$ обмотка отсутствует и $f_{1 y}=0$.

Токи лобовых частей обмотки имеют в различных областях активной зоны при $y=a$ следующие величины линейных плотностей $f_{1 x}$ : при $0 \leqslant x \leqslant g \beta \tau$

$$
J_{1 x \mathrm{H}}=\int_{0}^{x} J_{1 y \mathrm{H}} \mathrm{d} x
$$

при $g \beta \tau \leqslant x \leqslant 2 p \tau$

$$
f_{1 x \mathrm{c}}=\left.J_{1 x \mathrm{H}}\right|_{x=g \beta \tau}+\int_{g \beta \tau}^{x} J_{1 y c} \mathrm{~d} x
$$

и при $2 p \tau \leqslant x \leqslant 2 p \tau+g \beta \tau$

$$
J_{1 x \kappa}=\left.J_{1 x c}\right|_{x=2 p \tau}+\int_{2 p \tau}^{x} J_{1 y \kappa} \mathrm{d} \dot{x} .
$$

Подставляя в каждое полученное соотношение значения $f_{1 y}$ из выражений (1), (2) и (3) соответственно и интегрируя, получим

$$
\begin{gathered}
f_{1 x \mathrm{H}}=-\frac{i g f_{1 m}}{\alpha}\left(1-\mathrm{e}^{-i \alpha x}\right), \\
f_{1 x c}=\frac{i J_{1 m}}{\alpha}\left[\left(1-\mathrm{e}^{i \beta \pi}\right)\left(\mathrm{e}^{-i \alpha x}-\mathrm{e}^{-i g \beta \pi}\right)-g\left(1-\mathrm{e}^{-i g \beta \pi}\right)\right], \\
J_{1 x \mathrm{~K}}=\frac{i J_{1 m}}{\alpha}\left(1-\mathrm{e}^{i \beta \pi}\right)\left(\mathrm{e}^{-i 2 p \pi}-\mathrm{e}^{-i g \beta \pi}\right)- \\
-\frac{i g f_{1 m}}{\alpha}\left[1-\mathrm{e}^{-i g \beta \pi}+\mathrm{e}^{i \beta \pi}\left(\mathrm{e}^{-i \alpha \dot{x}}-\mathrm{e}^{-i 2 p \pi}\right)\right] .
\end{gathered}
$$




\section{Уравнения поля в зазоре}

В рассматриваемой задаче $\mathbf{H}$ имеет составляющую только по оси $z$ и поэтому, согласно $\left[{ }^{3}\right]$, для зоны а действительно уравнение

$$
\frac{\partial^{2} \dot{H}_{\mathrm{a}}}{\partial x^{2}}+\frac{\partial^{2} \dot{H}_{\mathrm{a}}}{\partial y^{2}}-2 \alpha \eta \frac{\partial \dot{H}_{\mathrm{a}}}{\partial x}-i \alpha^{2} \varepsilon_{0} \dot{H}_{\mathrm{a}}=\dot{N},
$$

где $\varepsilon_{0}=\mu_{0} \gamma \omega / \alpha^{2}-$ добротность машины, $\eta=\mu_{0} \gamma u / 2 \alpha, \omega-$ угловая частота, $u$ - скорость движения вторичной шины,

$$
\dot{N}=-\operatorname{rot}_{z} J_{1 y}=-\left(\frac{\partial J_{1 y}}{\partial x}-\frac{\partial J_{1 x}}{\partial y}\right)=-\frac{\partial J_{1 y}}{\partial x} .
$$

Значения $\dot{N}$ для различных зон можно вычислить по соотношениям (1) - (3) .

В зоне в $f_{1 y}=0, \dot{N}=0$ и поэтому, согласно изложенному выше,

$$
\frac{\partial^{2} \dot{H}_{\mathrm{B}}}{\partial x^{2}}+\frac{\partial^{2} \grave{H}_{\mathrm{B}}}{\partial y^{2}}=0
$$

Граничные условия. Вследствие симметрии задачи относительно оси $y$ можно рассматривать только область $y \geqslant 0$. Первое граничное условие при $y=0$ имеет вид

$$
\frac{\partial \dot{H}_{\mathrm{a}}}{\partial y}=0
$$

При $y=t$ составляющая вторичной плотности тока $f_{2 y}=0$ и, следовательно, $\operatorname{rot}_{y} \dot{H}_{\mathrm{B}}=-\frac{\partial \dot{H}_{\mathrm{B}}}{\partial x}=0$, а поэтому $\dot{H}_{\mathrm{B}}=$ const и по физическим соображениям

$$
\dot{H}_{\mathrm{B}}=0 .
$$

Исходя из закона полного тока, при $y=a$ можем записать

$$
\dot{H}_{\mathrm{B}}-\dot{H}_{\mathrm{a}}=J_{1 x} \text {. }
$$

Из условия равенства тангенциальных составляющих напряженности электрического поля и векторов плотности вторичного тока на границе раздела зон а и в при $y=a$ следует

$$
\frac{\partial \dot{H}_{\mathrm{a}}}{\partial y}=\frac{\partial \dot{H}_{\mathrm{B}}}{\partial y} .
$$

Р ешени е у р а вн ен и й поля. Используя метод интегрального преобразования Фурье $\left[{ }^{5,6}\right]$, находим изображение $H_{\mathrm{a}}(k)$ для $\dot{H}_{\mathrm{a}}$ по оси $x$ :

$$
H_{\mathrm{a}}(k)=\int_{-\infty}^{\infty} \dot{H}_{\mathrm{a}}(x, y) \mathrm{e}^{-i k x} \mathrm{~d} x .
$$

Согласно (15) из выражений (8) и (10) получим уравнения для изображений:

$$
\frac{\partial^{2} H_{\mathrm{a}}(k)}{\partial y^{2}}-\mathrm{Q}^{2} H_{\mathrm{a}}(k)=N(k),
$$




$$
\frac{\partial^{2} H_{\mathrm{B}}(k)}{\partial y^{2}}-k^{2} H_{\mathrm{B}}(k)=0,
$$

где

$$
\begin{gathered}
N(k)=\int_{-\infty}^{\infty} N \mathrm{e}^{-i k x} \mathrm{~d} x=\frac{-k J_{1 m}}{k+\alpha}\left[g+\mathrm{e}^{-i(k+\alpha) g \beta \tau}\left(1-\mathrm{e}^{i \beta \pi}-g\right)-\right. \\
\left.-\mathrm{e}^{-i(k+\alpha) 2 p \tau}\left(1-\mathrm{e}^{i \beta \pi}+g \mathrm{e}^{i \beta \pi}\right)+g \mathrm{e}^{i \beta \pi} \mathrm{e}^{-i(k+\alpha)(2 p \tau+g \beta \tau)}\right], \\
\mathrm{Q}^{2}=k^{2}+2 i \alpha \eta k+i \alpha^{2} \varepsilon_{0} .
\end{gathered}
$$

Учитывая граничные условия (11) и (12), решения уравнений (16) и (17) можем записать в виде

$$
\begin{aligned}
& H_{\mathrm{a}}(k)=C_{\mathrm{a}} \operatorname{ch} \mathrm{Q} y+H_{\mathrm{aq}}(k), \\
& H_{\mathrm{B}}(k)=C_{\mathrm{B}} \operatorname{sh} k(t-y),
\end{aligned}
$$

где

$$
H_{\text {aч }}(k)=-\frac{N(k)}{\varrho^{2}} .
$$

Постоянные интегрирования $C_{\mathrm{a}}$ и $C_{\mathrm{B}}$ определяются исходя из граничных условий (13) и (14) после выполнения над ними интегрального преобразования Фурье по $x$ :

при $y=a$.

$$
H_{\mathrm{B}}(k)-H_{\mathrm{a}}(k)=J_{1 x}(k)
$$

и

$$
\frac{\partial H_{\mathrm{a}}(k)}{\partial y}=\frac{\partial H_{\mathrm{B}}(k)}{\partial y}
$$

где

$$
J_{1 x}(k)=\int_{-\infty}^{\infty} J_{1 x} \mathrm{e}^{-i k x} \mathrm{~d} x
$$

После подстановки $f_{1 x}$ из выражений $(5)-(7)$ и интегрирования имеем

$$
\begin{gathered}
J_{1 x}(k)=\frac{J_{1 m}}{\alpha k(k+\alpha)}\left\{\mathrm{e}^{-i k g \beta \tau} \alpha \mathrm{e}^{-i g \beta \pi}\left(g-1+\mathrm{e}^{i \beta \pi}\right)+\mathrm{e}^{-i k 2 p \tau} \alpha \mathrm{e}^{-i 2 p \pi}\left(g \mathrm{e}^{i \beta \pi}+\right.\right. \\
\left.+1-\mathrm{e}^{i \beta \pi}\right)+\mathrm{e}^{-i k(2 p \tau+g \beta \tau)}\left[g(k+\alpha)-\mathrm{e}^{-i 2 p \pi}\left[(k+\alpha)\left(\mathrm{e}^{i \beta \pi}-1-g \mathrm{e}^{i \beta \pi}\right)+\right.\right. \\
\left.\left.\left.+k g \mathrm{e}^{i \beta \pi} \mathrm{e}^{-i g \beta \pi}\right]+(k+\alpha) \mathrm{e}^{-i g \beta \pi}\left(1-\mathrm{e}^{i \beta \pi}-g\right)\right]-g \alpha\right\} .
\end{gathered}
$$

На основании $(20),(21),(23)$ и $(24)$ находим

$$
\begin{aligned}
& C_{\mathrm{a}}=-\frac{\left[J_{1 x}(k)+H_{\mathrm{aq}}(k)\right] k \operatorname{ch} k(t-a)}{D(k)}, \\
& C_{\mathrm{B}}=\frac{\left[J_{1 x}(k)+H_{\mathrm{aq}}(k)\right] \varrho \operatorname{sh} \varrho a}{D(k)},
\end{aligned}
$$

где 


$$
D(k)=\varrho \operatorname{sh} \varrho a \operatorname{sh} k(t-a)+k \operatorname{ch} \varrho a \operatorname{ch} k(t-a) .
$$

C учетом $(20)-(22)$ и $(25)-(27)$ изображения Фурье для напряженностей магнитного поля в зонах а и в окончательно примут вид

$$
H_{\mathrm{a}}(k)=\frac{J_{1 m}\left[G_{0}(k, y)+G_{1}(k, y) \mathrm{e}^{-i k g \beta \tau}+G_{2}(k, y) \mathrm{e}^{-i k 2 p \tau}+G_{3}(k, y) \mathrm{e}^{-i k(2 p \tau+g \beta \tau)}\right]}{\mathrm{Q}^{2}(k+\alpha) D(k)},
$$

$$
H_{\mathrm{B}}(k)=\frac{J_{1 m}\left[\lambda_{1}(k) \mathrm{e}^{-i k g \beta \tau}+\lambda_{2}(k) \mathrm{e}^{-i k 2 p \tau}+\lambda_{3}(k) \mathrm{e}^{-i k(2 p \tau+g \beta \tau)}-\lambda_{0}(k)\right] M(k, y)}{k Q^{2}(k+\alpha) D(k)},
$$

где в случае однослойной обмотки индуктора (при $g=0$ и $\beta=1$ )

$G_{0}(k, y)=G_{3}(k, y)=0 ; \quad G_{1}(k, y)=-G_{2}(k, y)=2\left[k D(k)+\left(\varrho^{2}-k^{2}\right) T(k, y)\right] ;$ $\lambda_{0}(k)=\lambda_{3}(k)=0$; $\lambda_{1}(k)=-\lambda_{2}(k)=-2\left(\varrho^{2}-k^{2}\right)$;

в случае двуслойной (при $g=1$ и $\beta \neq 1$ )

$G_{0}(k, y)=-G_{1}(k, y)=k D(k)+\left(\varrho^{2}-k^{2}\right) T(k, y) ; \quad G_{2}(k, y)=-G_{3}(k, y)=$ $=G_{1}(k, y) \mathrm{e}^{-i 2 p \pi} ; \quad \lambda_{0}(k)=\lambda_{1}(k)=\mathrm{Q}^{2}-k^{2} ; \quad \lambda_{2}(k)=-\lambda_{3}(k)=\lambda_{1}(k) \mathrm{e}^{-i 2 p \pi}$, a $T(k, y)=\operatorname{ch} k(t-a) \operatorname{ch} \varrho y \quad$ и $\quad M(k, y)=\varrho \operatorname{sh} \varrho a \operatorname{sh} k(t-y)$.

Для получения оригиналов искомых функций применяется обратное преобразование Фурье $\left[{ }^{5}\right]$ :

$$
\begin{gathered}
{\left[G_{0}(k, y) \mathrm{e}^{i k x}+G_{1}(k, y) \mathrm{e}^{i k(x-g \beta \tau)}+\right.} \\
\dot{H}_{\mathrm{a}}=\frac{J_{1 m}}{2 \pi} \int_{-\infty}^{\infty} \frac{\left.+G_{2}(k, y) \mathrm{e}^{i k(x-2 p \tau)}+G_{3}(k, y) \mathrm{e}^{i k(x-2 p \tau-g \beta \tau)}\right]}{(k+\alpha) \mathrm{Q}^{2} D(k)} \cdot \mathrm{d}, \\
\dot{H}_{\mathrm{B}}=\frac{J_{1 m}}{2 \pi} \int_{-\infty}^{\infty} \frac{\left[\lambda_{1}(k) \mathrm{e}^{-i k g \beta \tau}+\lambda_{2}(k) \mathrm{e}^{-i k 2 p \tau}+\right.}{\left.k \lambda^{2}(k) \mathrm{e}^{-i k(2 p \tau+g \beta \tau)}-\lambda_{0}(k)\right] M(k, y)} \\
k \mathrm{Q}^{2}(k+\alpha) D(k)
\end{gathered}
$$

Интегрирование (31) и (32) выполняется с помощью теории вычетов. При этом необходимо определить полюсы подынтегральной функции, которые соответствуют корням знаменателя этой функции, приравненного к нулю. Можно показать, что корни $k \mathrm{Q}^{2}=0$ соответствуют устранимым особым точкам подынтегральной функции и поэтому нет необходимости их отыскивать. Один из корней остающихся частей знаменателей данных функций, очевидно,

$$
k=-\alpha,
$$

а остальные определяются из уравнения

$$
D(k)=0,
$$

которое имеет бесконечное количество корней. Аналитическое выражение для этих корней в общем случае получить невозможно, поэтому для их нахождения необходимо воспользоваться одним из численных методов $\left[{ }^{7}\right]$. Наибольший вклад в решение дают корни, амплитуды которых невелики, т. е. корни, расположенные близко от начала координат комплексной плоскости, так как затухание экспоненциальных чле- 
нов происходит тем медленнее, чем меньше показатель степени экспоненты.

В первом приближении можно ограничиться учетом первых членов разложения в ряд Тейлора гиперболических функций в (28), и тогда вместо уравнения (34) на основании (19) получим

$$
k(t-a) a\left(k^{2}+2 i a \eta k+i \alpha^{2} \varepsilon_{0}\right)+k=0 .
$$

Уравнение (35) имеет корни (рис. 3) $k=0$ и

$$
k_{11,21}=i\left(-\alpha \eta \pm \sqrt{(\alpha \eta)^{2}+1 / a(t-a)+i \alpha^{2} \varepsilon_{0}}\right) .
$$

При необходимости значения этих корней можно уточнить с помощью ЭВМ. Расчеты на ЭВМ показывают, что во многих случаях достаточно ограничиться учетом первой пары корней.

Если $x<0$, интеграл (31) берется как сумма вычетов в особых точках нижней полуплоскости $\left[{ }^{8}\right]$, и в общем случае:

$$
\dot{H}_{\mathrm{a}}=-2 \pi i \sum_{n=1}^{\infty} \operatorname{Res} H_{\mathrm{a}}\left(k_{2 n}\right)-\pi i\left[\operatorname{Res} H_{\mathrm{a}}(0)+\operatorname{Res} H_{\mathrm{a}}(-\alpha)\right] .
$$

При $0<x<g \beta \tau$ для члена, содержащего $\mathrm{e}^{i k x}$, берется сумма вычетов в верхней полуплоскости, а для всех остальных членов - в нижней полуплоскости:

$$
\begin{gathered}
\dot{H}_{\mathrm{a}}=2 \pi i \sum_{n=1}^{\infty}\left[\operatorname{Res} H_{\mathrm{a}}\left(k_{1 n}\right)-\operatorname{Res} H_{\mathrm{a}}\left(k_{2 n}\right)\right]+\pi i\left[\operatorname{Res}^{\mathrm{B}} H_{\mathrm{a}}(-\alpha)-\right. \\
\left.-\operatorname{Res}^{\text {म }} H_{\mathrm{a}}(-\alpha)+\operatorname{Res}^{\mathrm{B}} H_{\mathrm{a}}(0)-\operatorname{Res}^{\mathrm{H}} H_{\mathrm{a}}(0)\right],
\end{gathered}
$$

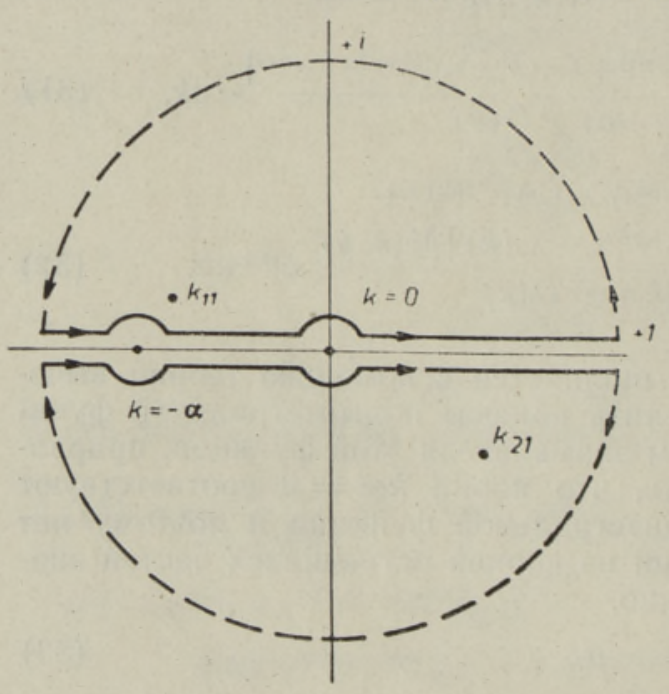

Рис. 3. Расположение полюсов подынтегральной функции в комплексной плоскости. где $n$ - число пар комплексных корней (34).

В случае $g \beta \tau<x<2 p \tau$ члены с $\mathrm{e}^{i k x}$ и $\mathrm{e}^{i k(x-g \beta \tau)}$ интегрируем по верхней полуокружности, остальные - по нижней; при $2 p \tau<x<2 p \tau+g \beta \tau$ вычеты для члена с $\mathrm{e}^{i k(x-2 p \tau-g \beta x)}$ берутся в нижней полуплоскости, для остальных членов - в верхней. Для этих двух зон $\dot{H}_{\mathrm{a}}$ определяется также по схеме (38). Если $x>2 p \tau+g \beta \tau$, то ннтегрирование (31) полностью производится по верхней полуокружностн.

В итоге для напряженности магнитного поля имеем: при $x<0$

$$
\begin{gathered}
\dot{H}_{\mathrm{a}}=i J_{1 m} \sum_{n=1}^{\infty}\left[\left(\lambda_{1}\left(k_{2 n}\right) \mathrm{e}^{-i k_{2 n} g \beta \tau}+\lambda_{2}\left(k_{2 n}\right) \mathrm{e}^{-i k_{2 n} 2 p \tau}+\right.\right. \\
\left.\left.+\lambda_{3}\left(k_{2 n}\right) \mathrm{e}^{-i k_{2 n}(2 p \tau+g \beta \tau)}-\lambda_{0}\left(k_{2 n}\right)\right) \frac{T\left(k_{2 n}, y\right) \mathrm{e}^{i k_{2 n} x}}{\left(k_{2 n}+\alpha\right) \varrho_{2 n}^{2} D^{\prime}\left(k_{2 n}\right)}\right],
\end{gathered}
$$


при $0<x<g \beta \tau$

$$
\begin{gathered}
\dot{H}_{\mathrm{a}}=i J_{1 m}\left\{\frac{G_{0}(-\alpha, y)}{\varrho_{-\alpha}^{2} D(-\alpha)} \mathrm{e}^{-i \alpha x}+\sum_{n=1}^{\infty}\left[\frac{\lambda_{0}\left(k_{1 n}\right) T\left(k_{1 n}, y\right) \mathrm{e}^{i k_{1 n} x}}{\left(k_{1 n}+\alpha\right) \mathrm{Q}_{1 n}^{2} D^{\prime}\left(k_{1 n}\right)}+\right.\right. \\
+\left(\lambda_{1}\left(k_{2 n}\right) \mathrm{e}^{-i k_{2 n} g \beta \tau}+\lambda_{2}\left(k_{2 n}\right) \mathrm{e}^{-i k_{2 n} 2 p \tau}+\lambda_{3}\left(k_{2 n}\right) \mathrm{e}^{-i k_{2 n}(2 p \tau+g \beta \tau)}\right) \times \\
\left.\left.\times \frac{T\left(k_{2 n}, y\right) \mathrm{e}^{i k_{2 n} x}}{\left(k_{2 n}+\alpha\right) \varrho_{2 n}^{2} D^{\prime}\left(k_{2 n}\right)}\right]+\frac{G_{0}(0, y)}{\alpha \varrho_{0}^{2} D^{\prime}(0)}\right\},
\end{gathered}
$$

при $g \beta \tau<x<2 p \tau$

$$
\begin{gathered}
\dot{H}_{\mathrm{a}}=i J_{1 m}\left\{\frac{G_{0}(-\alpha, y)+G_{1}(-\alpha, y) \mathrm{e}^{i g \beta \pi}}{\mathrm{Q}_{-\alpha}^{2} D(-\alpha)} \mathrm{e}^{-i \alpha x}+\sum_{n=1}^{\infty}\left[\left(\lambda_{0}\left(k_{1 n}\right)-\right.\right.\right. \\
\left.-\lambda_{1}\left(k_{1 n}\right) \mathrm{e}^{-i k_{n n} g \beta \tau}\right) \frac{T\left(k_{1 n}, y\right) \mathrm{e}^{i k_{1 n} x}}{\left(k_{1 n}+\alpha\right) Q_{1 n}^{2} D^{\prime}\left(k_{1 n}\right)}+\left(\lambda_{2}\left(k_{2 n}\right) \mathrm{e}^{-i k_{2 n} 2 p \tau}+\right. \\
\left.\left.\left.+\lambda_{3}\left(k_{2 n}\right) \mathrm{e}^{-i k_{2 n}(2 p \tau+g \beta \tau)}\right) \frac{T\left(k_{2 n}, y\right) e^{i k_{2 n} x}}{\left(k_{2 n}+\alpha\right) Q_{2 n}^{2} D^{\prime}\left(k_{2 n}\right)}\right]+\frac{G_{0}(0, y)+G_{1}(0, y)}{\alpha \varrho_{0}^{2} D^{\prime}(0)}\right\},
\end{gathered}
$$

при $2 p \tau<x<2 p \tau+g \beta \tau$

$$
\begin{aligned}
& \dot{H}_{\mathrm{a}}=i J_{1 m}\left\{\frac{-G_{3}(-\alpha, y) \mathrm{e}^{i(2 p \pi+g \beta \pi)}}{\mathrm{Q}_{-\alpha}^{2} D(-\alpha)} \mathrm{e}^{-i \alpha x}+\sum_{n=1}^{\infty}\left[\left(\lambda_{0}\left(k_{1 n}\right)-\right.\right.\right. \\
& \left.-\lambda_{1}\left(k_{1 n}\right) \mathrm{e}^{-i k_{1 n} g \beta \tau}-\lambda_{2}\left(k_{1 n}\right) \mathrm{e}^{-i k_{1 n}{ }^{2} p \tau}\right) \frac{T\left(k_{1 n}, y\right) \mathrm{e}^{i k_{1 n} x}}{\left(k_{1 n}+\alpha\right) \mathrm{Q}_{1 n}^{2} D^{\prime}\left(k_{1 n}\right)}+ \\
& \left.\left.+\lambda_{3}\left(k_{2 n}\right) \mathrm{e}^{-i k_{2 n}(2 p \tau+g \beta \tau)} \frac{T\left(k_{2 n}, y\right) \mathrm{e}^{i k_{2 n} x}}{\left(k_{2 n}+\alpha\right) \varrho_{2 n}^{2} D^{\prime}\left(k_{2 n}\right)}\right]-\frac{G_{3}(0, y)}{\alpha \varrho_{0}^{2} D^{\prime}(0)}\right\},
\end{aligned}
$$

при $2 p \tau+g \beta \tau<x<+\infty$

$$
\begin{gathered}
\dot{H}_{\mathrm{a}}=i J_{1 m} \sum_{n=1}^{\infty}\left[\left(\lambda_{0}\left(k_{1 n}\right)-\lambda_{1}\left(k_{1 n}\right) \mathrm{e}^{-i k_{1 n} g \beta \tau}-\lambda_{2}\left(k_{1 n}\right) \mathrm{e}^{-i k_{1 n} 2 p \tau}-\right.\right. \\
\left.\left.-\lambda_{3}\left(k_{1 n}\right) \mathrm{e}^{-i k_{1 n}(2 p \tau+g \beta \tau)}\right) \frac{T\left(k_{1 n}, y\right) \mathrm{e}^{i k_{1 n} x}}{\left(k_{1 n}+\alpha\right) \mathrm{Q}_{1 n}^{2} D^{\prime}\left(k_{1 n}\right)}\right],
\end{gathered}
$$

где

$$
D^{\prime}(k)=\operatorname{ch} \varrho a \operatorname{sh} k(t-a)[a(k+i \alpha \eta)+k(t-a)]+\operatorname{ch} \varrho a \operatorname{ch} k(t-a)+
$$$$
+\operatorname{sh} \varrho a \operatorname{ch} k(t-a)\left[\varrho(t-a)+k a \frac{k+i \alpha \eta}{\varrho}\right]+\frac{k+i \alpha \eta}{\varrho} \operatorname{sh} \varrho a \operatorname{sh} k(t-a) \text {, }
$$

$$
\mathrm{Q}_{1 n} \text { и } \mathrm{Q}_{2 n} \text { - значения @ при } k=k_{1 n} \text { и } k=k_{2 n} \text { соответственно. }
$$

Аналитические выражения для $\dot{H}_{\text {в }}$ можно найти, интегрируя аналогичным способом правую часть равенства (32).

Зависимости $(40)-(42)$ определяют напряженность магнитного поля в активной зоне ЛАМ и, если $2 p$ четное число, справедливы для машин как с двуслойными, так и с однослойными обмотками, в противном случае ( $2 p$ нечетное) - только для ЛАМ с двуслойными обмотками. 
Здесь член, содержащий $\mathrm{e}^{-i \alpha x}$, представляет собой нормальную бегущую волну $\dot{H}$, члены с $\mathrm{e}^{i k_{1 n} x}$ и $\mathrm{e}^{i k_{2 n} x}-$ соответственно прямо и обратно бегущие волны краевых эффектов, а последний член - пульсирующую составляющую $\dot{H}$, зависящую только от параметров вторичной среды, частоты первичного тока, величины полюсного деления и поперечных размеров машины.

Определение на основании вышеизложенной теории таких интегральных электромагнитных величин, как мощность ЛАМ, тяговое усилие и т. д., и их анализ предполагается изложить в следующей статье.

\section{Л И ТЕР А Т У Р А}

1. O b e r r et 1, K., Arch. Elektrotechn., 55, H. 4, 181 (1973).

2. O b e r r et l, K., A n c e 1, I., Rev. Gén. Electr., 85, N. 3, 331 (1976).

3. В ольдек А. И., Индукционные магнитогидродинамические машины с жидкометаллическим рабочим телом, Л., 1970.

4. B olt o n, H., Proc. Instn. Electr. Engrs., 116, No. 5, 725 (1969).

5. А н го А., Математика для электро- и радиоинженеров, М., 1964.

6. Y a m a mura, S., I t o, H., I sh ik a wa, Y., IEEE Trans. PAS., 91, No. 4, 1700 (1972).

7. Л ан с Дж. Н., Численные методы для быстродействующих вычислительных машин, М., 1962.

8. Ш.в а рц Л., Анализ, т. 2, М., 1972.

Ленинградский политехнический институт им. М. Н. Калинина

Поступила в редакцию 1/II 1977

A. VOLDEK , G. SKRJABINA

\section{PIKI- JA RISTÄAREEFEKTI ARVESTAV LINEAARSE ASUNKROONMASINA TEOORIA}

Fourier' integraalmuutujate meetodit rakendades on esitatud elektromagnetvälja jaotus lineaarsete asünkroonmasinate ōhupilus, kusjuures on arvestatud laupühenduste voolukatet ning piki- ja ristääreefekti üheaegset môju.

A. VOLDEK , G. SKRYABINA

\section{THE THEORY OF LINEAR INDUCTION MACHINE WITH LONGITUDINAL END AND TRANSVERSE EDGE EFFECTS}

In this paper the authors treat a problem about the distribution of electromagnetic field at the air gap in the linear induction machine, using Fourier transforms. The currents of the end parts of windings as well as the half-filled end slots of primary core are taken into account. A theory is developed that the longitudinal end effect and the transverse edge effect must be taken into account. 